\title{
Rhizosphere-associated bacterial network structure and spatial distribution differ significantly from bulk soil in wheat crop fields
}

\author{
Kunkun Fan $^{\text {a, b }}$, Cesar Cardona ${ }^{\text {c, d }}$, Yuntao Li ${ }^{\text {a, b }}$, Yu Shi ${ }^{\text {a }}$, Xingjia Xiang a, b, \\ Congcong Shen ${ }^{a, e}$, Hongfei Wang a , Jack A. Gilbert ${ }^{\mathrm{d}, \mathrm{f}, \mathrm{g}}$, Haiyan $\mathrm{Chu}^{\text {a, * }}$ \\ ${ }^{a}$ State Key Laboratory of Soil and Sustainable Agriculture, Institute of Soil Science, Chinese Academy of Sciences, 71 East Beijing Road, Nanjing 210008, \\ China \\ ${ }^{\mathrm{b}}$ University of Chinese Academy of Sciences, Beijing 100049, China \\ ${ }^{c}$ Graduate Program in Biophysical Sciences, University of Chicago, Chicago, IL 60637, USA \\ d Department of Surgery, University of Chicago, Chicago, IL 60637, USA \\ e Research Center for Eco-Environmental Sciences, Chinese Academy of Sciences, Beijing, 100085, China \\ ${ }^{\mathrm{f}}$ Biosciences Division, The Microbiome Center, Argonne National Laboratory, Lemont, IL 60439, USA \\ ${ }^{\mathrm{g}}$ Marine Biological Laboratory, Woods Hole, MA 02543, USA
}

\section{A R T I C L E I N F O}

\section{Article history:}

Received 8 February 2017

Received in revised form 5 June 2017

Accepted 18 June 2017

Available online 26 June 2017

\section{Keywords:}

Network

Bacterial community

Distribution

Rhizosphere

\begin{abstract}
A B S T R A C T
The spatial distribution of bacteria in bulk soil has been well studied, but little is known about the bacterial biogeography in the rhizosphere of crops. Here, we investigated bacterial distribution in bulk soil, loosely- and tightly-bound soils, from wheat fields distributed across $800,000 \mathrm{~km}^{2}$ of the North China Plain. Bacterial community composition differed dramatically among bulk and rhizospheric soils, and bacterial diversity decreased with the root proximity. Soil pH correlated with bacterial community composition and diversity in three compartments. Bacterial community in tightly bound soil formed a hub-based network topology with higher transitivity and greater number of central nodes compared with loosely bound and bulk soils, potentially as a result of more direct ecological interactions between the members of the tightly bound soil compartment. Bulk and rhizospheric soils maintained similar compositional distance decay patterns (with equal decay rates), but distinct phylogenetic distance decay patterns (with steeper slope of tightly bound soil). Geographical distance described a relatively greater proportion of bacterial spatial distribution in tightly bound soil, compared with loosely bound soil and bulk soil. Deterministic processes dominated the assemblage of bacterial communities in all soil compartments, while phylogenetic clustering was weaker in tightly bound soil. Taken together, our results suggest distinct bacterial network structure and distribution patterns among bulk soil, loosely bound soil and tightly bound rhizospheric soil, which could possibly result in potential functional differentiation.
\end{abstract}

(C) 2017 Elsevier Ltd. All rights reserved.

\section{Introduction}

Soil bacterial distribution has been widely investigated in natural ecosystems (Fierer and Jackson, 2006) and agricultural ecosystem (Carlson et al., 2012; Gumiere et al., 2016), however, bacterial biogeographic patterns in the rhizosphere of crop systems remain relatively unexplored. Soil $\mathrm{pH}$ correlates with bacterial distribution in many ecosystems, including forests (Nacke et al., 2011), grasslands (Will et al., 2010) and tundra (Chu et al., 2010; Shen et al., 2013). However, soil carbon content (Chu et al., 2016),

\footnotetext{
* Corresponding author.

E-mail address: hychu@issas.ac.cn (H. Chu).
}

soil carbon to nitrogen ratio (Högberg et al., 2007) and temperature (Zhou et al., 2016) also correlate with the distribution of bacterial communities in certain ecosystems. These differences may be in part due to the different study designs and methods used to generate the microbial profiles, and historical contingencies or spatial distances have also been shown to influence the distribution of microbial community at regional (Griffiths et al., 2011), continental (Martiny et al., 2011), and global (Caruso et al., 2011) scales. Agricultural ecosystems are often more homogeneous across spatial scales, with lower plant diversity and frequent human disturbance when compared to natural environments (Kennedy and Smith, 1995). While soil pH has been identified as a key factor correlating with microbial communities in agricultural soils in 
China (Liu et al., 2014, 2016), the type of history of agricultural activity for a given site has been shown to describe a greater degree of variance in microbial distribution when compared to $\mathrm{pH}$ ( $\mathrm{Ge}$ et al., 2008). Importantly, while the biogeography of bulk agricultural soil has been extensively investigated (Ge et al., 2008; Liu et al., 2014), the factors driving bacterial community distribution patterns in rhizospheric soil remain relatively unknown (Bulgarelli et al., 2015).

The rhizosphere is a biological hotspot whose physicochemical properties differ substantially from the surrounding bulk soil. Root exudation of low-molecular-weight organic acids can fundamentally alter the biogeochemistry of this environment (Wang et al., 2002). It is hypothesized that plants secrete these organic compounds to support microbial activity near the roots, which, in turn, can provide beneficial services to the plant, including enhanced mineral acquisition and pathogen protection (Dennis et al., 2010; Turner et al., 2013). Bacterial community composition has been shown to differ substantially among different rhizosphere compartments, with the diversity of these communities decreasing from the bulk soil towards the root (Donn et al., 2015). A similar pattern has also been observed in rice, which supports a multistep model for root microbiome assembly from soil (Edwards et al., 2015). In grapevines, the root and plant-tissue associated communities have been shown to be a subset of the bulk soil community (Zarraonaindia et al., 2015). A less complex network structure has also been found in rhizosphere when comparing with bulk soil in a short-term plantation system (Mendes et al., 2014). Most of these studies were limited to a small number of spatially proximal sites, or confined to a greenhouse or short-term plantation systems; therefore, the structures of root-associated compartments in typical farmland remain unclear.

Although the properties of the rhizosphere soil are modified by a range of processes occurring during plant growth (Philippot et al., 2013), distance decay relationships can still capture the variance in bacterial community composition and phylogeny across spatial scales. Microbial community structure is shaped mainly by deterministic factors (such as competition, niche differentiation) and neutral processes (Ofițeru et al., 2010). A recent short-term (1-5 years) soybean cultivation study demonstrated that microbial community selection in the rhizosphere occurred via niche filtering, while the bulk soil composition and structure seemed to be regulated by neutral processes (Mendes et al., 2014). Some studies have shown that both deterministic and neutral processes operate in structuring the same microbial community (Dumbrell et al., 2010; Caruso et al., 2011; Ferrenberg et al., 2013).

In this study, we combined bulk and rhizospheric soils to investigate bacterial distribution patterns in agricultural ecosystem. We further collected the soils from three compartments namely bulk soil, loosely bound soil and tightly bound soil according to the root proximity, and tightly bound soil was always regarded as rhizosphere soil (Donn et al., 2015). The sampling sites were located in wheat fields on the North China Plain, which is an important agricultural area in China, with a long-term (about 40 years) wheat-maize rotation system (Chen et al., 2004). Wheat (Triticumaestivum L.) is one of the main grain crops globally, but productivity increases per year have slowed to $0.9 \%$ (Fischer and Edmeades, 2010). It is possible that targeted manipulation of soil processes, including those involving microbial biogeochemistry, could help to accelerate crop productivity again. The rhizosphere is intriguingly complex and dynamic (Philippot et al., 2013), and understanding the microbial distribution patterns therein is key to developing strategies for enhancing plant productivity and ecosystem function (Brink, 2016). We addressed three hypotheses in the current study. First, the physicochemical properties that correlate with bacterial community structure are different in different compartments. Second, the network co-association properties within bacterial communities differ significantly between soil compartments. Third, the biogeographic distribution patterns across spatial distance (compositional and phylogenetic distance decay) and processes of bacterial community assembly vary among soil compartments.

\section{Materials and methods}

\subsection{Field survey and sample collection}

Wheat field survey data of North China Plain were collected from the Chinese agriculture web system (http://english.agri.gov. $\mathrm{cn} /$ ). We also used GIS (Geographic Information System) map to choose the sampling sites under same wheat-maize rotation system, and determine plot locations based on the following approach. Nine study sites, each $\sim 100 \mathrm{~km}^{2}$ square region, were identified across typical wheat planting fields over a broad area $\left(\sim 800,000 \mathrm{~km}^{2}\right)\left(32^{\circ} \mathrm{N} \sim 38^{\circ} \mathrm{N} ; 110^{\circ} \mathrm{E} \sim 118^{\circ} \mathrm{E}\right)$ of the North China Plain (Fig. S1A) during the wheat filling stage (Soils from the wheat field in the nine sites were collected on 22nd-28th of the May, 2015). Within each of the nine sampling sites, five locations (one in each corner and one in the center) were chosen for repeat sampling (Fig. S1B). At each of the five repeat locations, six groups of plants (four to six plants in each group) were extracted by digging around the groups to collect loosely bound soil and tightly bound soil (Donn et al., 2015). Plants were lightly shaken by holding with shoots to get the loosely bound soil and then soils remain attached to the root surface were brushed down as tightly bound soil which was defined as rhizosphere soil (Philippot et al., 2013). The topsoil $(0-15 \mathrm{~cm})$, beside each group $\sim 50 \mathrm{~cm}$ without plants, were collected by drill as bulk soil (BS). At each sampling time, the drill and brush were washed with sterile water and then air dried. Samples at each of the five repeat locations were pooled into a single composite sample for BS, LS and TS, respectively. All the samples were packed into polyethylene bags and immediately transported on ice packs to the laboratory. The soils were sieved through $2 \mathrm{~mm}$ meshes to remove visible roots, residues and stones. Each composite sample was then divided into two parts: one was stored at $4{ }^{\circ} \mathrm{C}$ for the soil biogeochemical properties analyses and the other one was stored at $-20^{\circ} \mathrm{C}$ for DNA extraction within two weeks.

\subsection{Soil chemical analysis}

There were 135 samples in total: 45 samples for each compartment (bulk soil, loosely bound soil and tightly bound soil). The soil type of most sampling locations were Typic Ochri-Aquic Cambosols and Typic Hapli-Udic Argosols; sandy loam soil and clay loam soil dominated the soil texture (Table S1). For each soil sample, soil moisture was measured for 12 times using QS-SFY, and soil $\mathrm{pH}$ was determined with a fresh soil to water ratio of $1: 5$ using a $\mathrm{pH}$ monitor (Thermo Orion-868). Soil was air dried and sieved (1 mm mesh), and total carbon (TC), total nitrogen (TN), total phosphorus (TP) and total potassium (TK) were determined by combustion (CNS-2000; LECO, St. Joseph, MI, USA).

\subsection{PCR amplification and high throughput sequencing}

A total of $0.5 \mathrm{~g}$ of fresh soil was used for DNA extraction using the Power Soil DNA kit (MO BIO Laboratories, Carlsbad, CA, USA) following the manufacturer's instructions. Primers $515 \mathrm{~F}\left(5^{\prime}-\right.$ GTGCCAGCMGCCGCGGTAA- ${ }^{\prime}$ ) and 907R (5'-CCGTCAATTCCTTTGAGTTT-3') (Biddle et al., 2008), which targeting the bacterial 16S rRNA V4-V5 region, were used to amplify the 16S rRNA gene 
fragment from each sample, and were sequenced on the Illumina MiSeq PE250 platform. PCR was performed in $50 \mu$ reactions containing $25 \mu \mathrm{l}$ Premix Taq DNA polymerase, $0.5 \mu \mathrm{l}$ forward primer $(20 \mu \mathrm{M}), 0.5 \mu \mathrm{l}$ reverse primer $(20 \mu \mathrm{M}), 23 \mu \mathrm{l}$ double distilled water $\left(\mathrm{ddH}_{2} \mathrm{O}\right)$, and $1 \mu \mathrm{l}$ DNA template (20 ng total soil DNA) under the conditions of $94^{\circ} \mathrm{C}$ for $5 \mathrm{~min}, 35$ cycles of $94^{\circ} \mathrm{Cfor} 30 \mathrm{~s}$, at $50{ }^{\circ} \mathrm{C}$ for $30 \mathrm{~s}$, and $72{ }^{\circ} \mathrm{C}$ for $30 \mathrm{~s}$. Sequences obtained from this research were submitted in the NCBI Sequence Read Archive (SRA) with accession number SRP093471.

\subsection{Bioinformatics and statistics}

After sequencing, the raw data were analyzed using the Quantitative Insight into Microbial Ecology (QIIME) pipeline (http:// qiime.sourceforge.net/) (Caporaso et al., 2010). Reads which were $<200$ bp long or had an average quality score $<25$ were removed (Huse et al., 2007), resulting in 5,595,603 high quality sequences. Clustering of quality sequences into operational taxonomic units (OTUs) was performed with UCLUST based on a $97 \%$ similarity level (Edgar, 2010). The taxonomic identity of each OTU was predicted based on similarity to the Green genes database (http://greengenes. lbl.gov/). The OTU richness is calculated as the observed number of OTUs in each sample, and the phylogenetic diversity (PD) index was used to determine the phylogenetic diversity.

SPSS20.0 was used to do the ANOVA, Pairwise $t$-test and to calculate the correlations between microbial diversity and soil variables. The Kruskal-Wallis test was calculated in the package “dplyr" R $\times 32$ (3.2.2) (http://cran.stat.sfu.ca/). Non-metric multidimensional scaling analyses (NMDS) were performed on the BrayCurtis dissimilarity for the microbial community data. The NMDS, SIMPER analysis, Mantel test, Canonical Correspondence Analysis (CCA), Partial CCA-based variation partitioning analysis and Partial mantel tests were conducted using the "vegan" package $\mathrm{R} \times 32$ (3.2.2). Multiple regression with distance matrices (MRM) was calculated in "ecodist" packages. Spiec-Easi (Sparse InversE Covariance Estimation for Ecological Association Inference) network analysis in both OTU level and class level (Kurtz et al., 2015; Cardona et al., 2016) which supported the mutual corroboration carried out in the R environment (http://www.r-project.org/) with "SpiecEasi" package, and the properties were calculated in "igraph" package. Distance decay curve of compositional similarity (1-braycurtis distance) was calculated (Nekola and White, 1999) while phylogenetic distance decay relationship (1-betaMNTD; 1 unweighted_Unifrac distance) was related to Bryant's analysis (Bryant et al., 2008) and calculated in R (Saito et al., 2015).

The net relatedness index (NRI) calculates the mean phylogenetic distance (MPDs) among all co-occurring individuals and it therefore indicates the 'basal' dispersion of the lineages within the community. The nearest taxon index (NTI) measures the mean nearest taxon distance (MNTD) among individuals and it therefore estimates the 'terminal' phylogenetic dispersion of the community (Webb, 2000). For a single community, NTI greater than +2 indicates coexisting taxa are more closely related than expected by chance (phylogenetic clustering). NTI less than -2 indicates coexisting taxa are more distantly related than expected by chance (phylogenetic over-dispersion). When mean NRI and NTI taken across all communities were above zero it suggests that communities are phylogenetically clustered, and if the mean values were negative, communities were phylogenetically over-dispersed. NRI and NTI were calculated in the R "picante" package (Purcell et al., 2007). Beta nearest taxon index (betaNTI) is the number of standard deviations that the observed betaMNTD is from the mean of null distribution, betaNTI below -2 or above +2 indicates less than or greater than expected phylogenetic turnover, for one pair-wise comparison (Stegen et al., 2012). The betaNTI was calculated in phylocom4.2 (Hardy, 2008).

\section{Results}

\subsection{Soil chemical properties in bulk soil and rhizospheric soil}

A pairwise $t$-test demonstrated that soil $\mathrm{pH}$ was significantly different in bulk soil, loosely bound soil and tightly bound soil in seven of nine sampling sites. The standard deviations within tightly bound soil in each sampling site were lower than that within loosely bound soil and bulk soil. The total potassium content in bulk soil and tightly bound soil was also significantly different in eight of the nine sampling sites. Four of the nine sampling sites had significantly different total carbon content between bulk soil and tightly bound soil. Soil total phosphorus content was not significantly different at any sampling site (Table S2).

\subsection{Bacterial community composition and diversity in bulk soil and rhizospheric soil}

In total 26,729 OTUs (97\% nucleotide identity) were clustered from 5,595,603 high-quality $16 \mathrm{~S}$ rRNA reads across all soil samples. Proteobacteria, Actinobacteria and Acidobacteria dominated all soils (Fig. 1A; Table S3). ANOVA identified Actinobacteria, Bacteroidetes, Alphaproteobacteria and Verrucomicrobia as being significantly more abundant in the tightly bound soil, compared to loosely bound soil and the bulk soil; while Gammaproteobacteria, Chloroflexi and Deltaproteobacteria were significantly less abundant in the tightly bound soil compared to the other soils. SIMPER analysis (Fig. 2) suggested that the variance between the bulk soil and loosely bound soil could mostly be described by changes in the abundance of Actinobacteria, while Bacteroidetes and Acidobacteria described variance in bulk soil and loosely bound soil when compared with tightly bound soil. We also used KruskalWallis test to compare the differences in family level pair-wise between compartments. Nocardioidaceae, Comamonadaceae, Hyphomicrobiaceae, Chitinophagaceae, Oxalobacteraceae, Sphingobacteriaceae, Streptomycetaceae and Microbacteriaceae were dominant families that were enriched in tightly bound soil compared with loosely bound soil and bulk soil. While Gaiellaceae, Rhodospirillaceae, Sinobacteraceae and Syntrophobacteraceae were dominant families which mostly depleted in tightly bound soil when comparing with loosely bound soil and bulk soil (Table S4). Bacterial alpha diversity was significantly correlated with distance to roots, so that samples in tightly bound soil had lower diversity than loosely bound $(\mathrm{P}=0.01$, ANOVA) or bulk soil $(\mathrm{P}=0.007$, ANOVA) (Fig. 1B; Table S5). Beta-diversity analysis using Bray-Curtis distance suggested that bulk soil, loosely bound soil and tightly bound soil each had a different microbial community structure (Fig. 2; Table S6).

\subsection{Environmental factors that correlate with bacterial diversity in bulk soil, loosely bound soil and tightly bound soil}

Mantel tests identified soil $\mathrm{pH}$ as the dominant factor correlating with microbial community structures (Table S7), which is self-evident from the NMDS visualization (Fig. 2). Matrix correlation analysis with bacterial community composition in phylum level (Table S8), family level (Table S9) and bacterial diversity (Table S10) confirms this relationship with soil pH. Partial CCAbased variation partitioning analysis also demonstrated that $\mathrm{pH}$ was less correlated with community structure in the tightly bound soil ( $4.3 \%$ of variance described by $\mathrm{pH})$, compared to loosely bound soil $(8.1 \%)$ and bulk soil (6.8\%) (Table S11). Total phosphorus was positively correlated with dominant bacteria (Table S8, Table S9) 


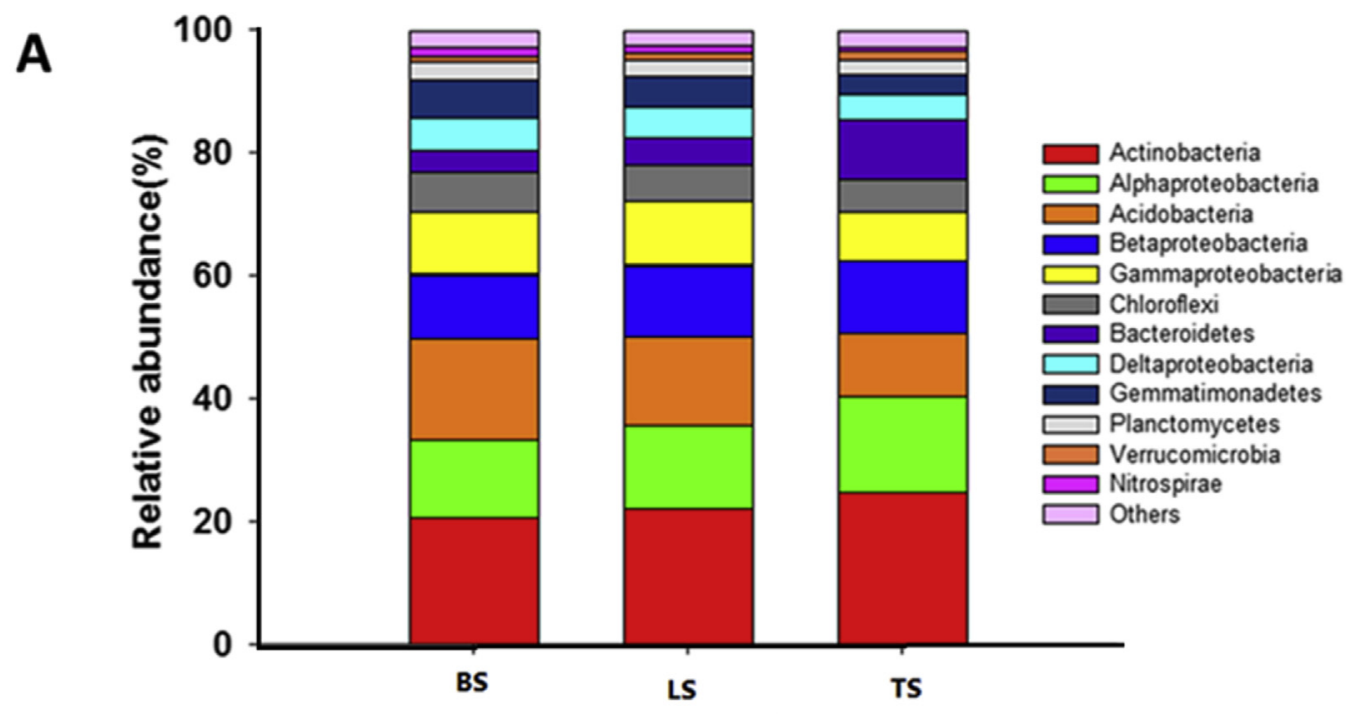

B
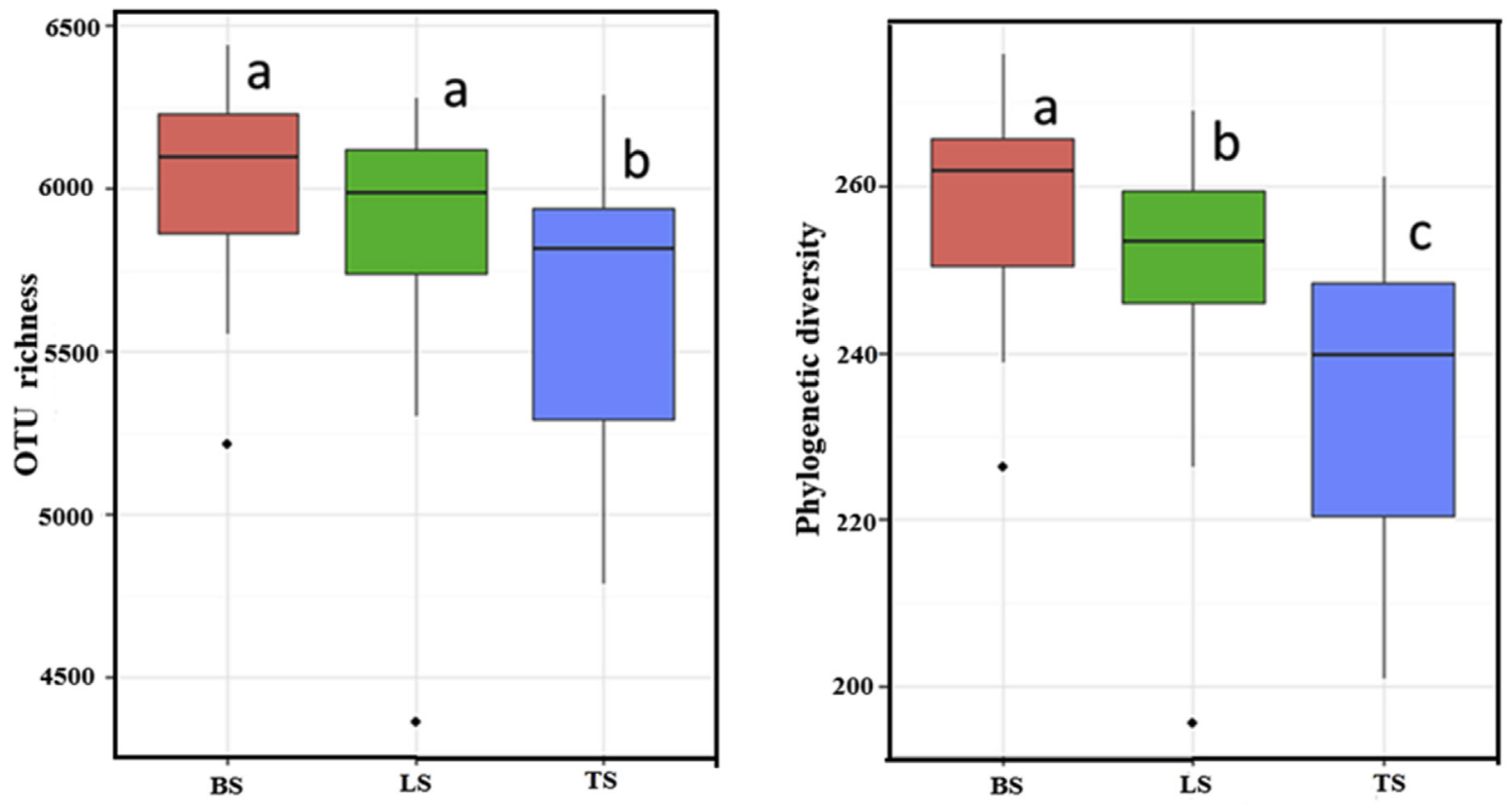

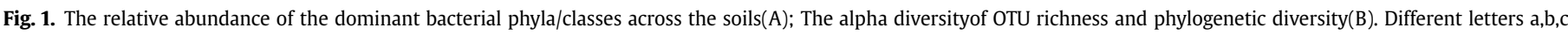
differ significantly at $\mathrm{P}<0.05$ (Duncan's test). BS:bulk soil; LS:loosely bound soil; TS:tightly bound soil.

and bacterial alpha diversity (Table S10), while soil moisture was negatively correlated with alpha diversity (Table S10). Other factors might also affect the community composition but not in a consistent and predictable way.

\subsection{Network topological features within bulk soil, loosely bound soil and tightly bound soil}

In order to explore the co-associated properties of microbial organisms in bulk soil, loosely bound soil and tightly bound soil, we employed Spiec-Easi network analysis at both the OTU (relevant abundance is more than $0.01 \%$ ) and the Class level, then calculated the network topological features of these networks in each soil compartment (Tables S12 and S13); the network map on Class level annotations demonstrates the topographical structure of these networks (Fig. 3A). The different soil compartment networks at the class level had the same number of nodes (177), but bulk soil had greater density (0.027), number of edges (424), degree (4.79), node betweeness centrality (202.6) and node closeness centrality (0.0003) than other soil compartments, which were approximately similar to the results of the network at the OTU level (Tables S13A and B). Class level features also show a partition of the overall network in several clusters, loosely bound soil had the greatest number of clusters (46), considerably greater than tightly bound soil (28) and bulk soil (13), each compartment had a single main sub-network (the largest cluster) and many others with a single or few elements, reflecting a high number of no co-occurrences across different classes. Nonetheless, each main sub-network showed dissimilar pattern among compartments (Table S13C). Tightly bound soil had the lowest degree (3.73), but the highest transitivity 


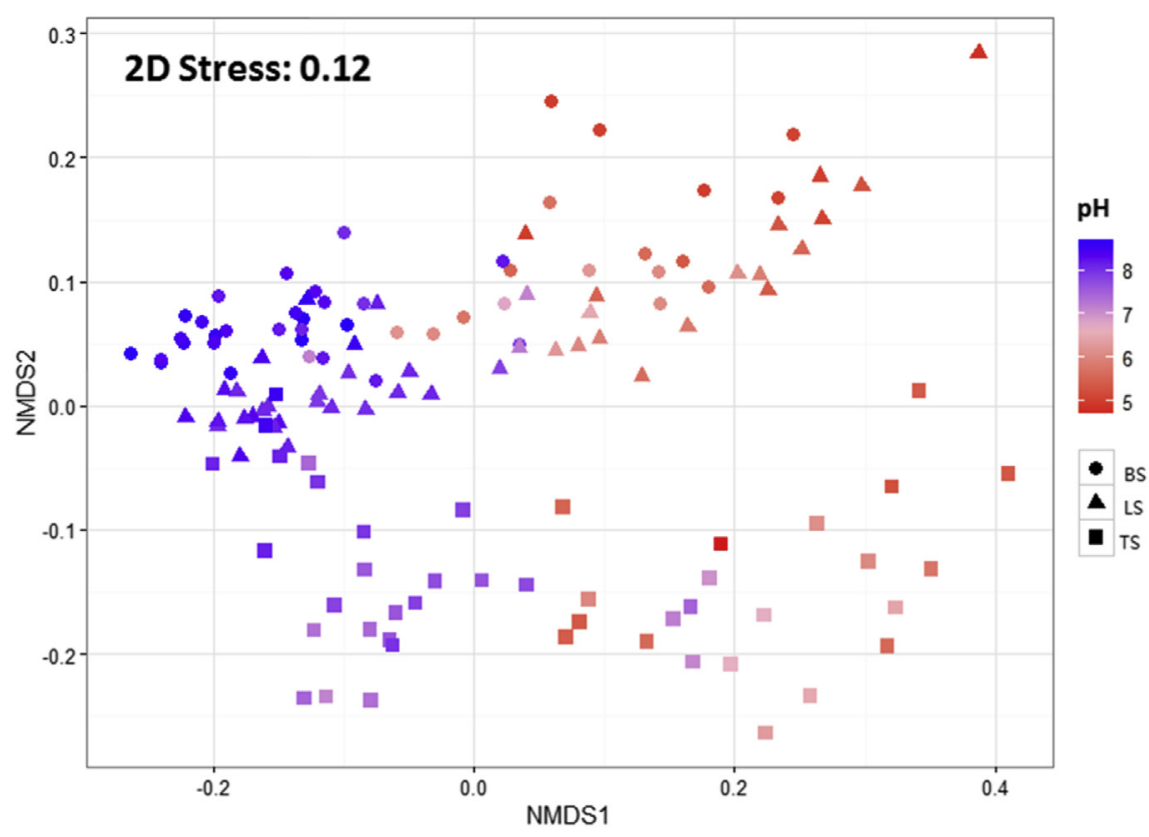

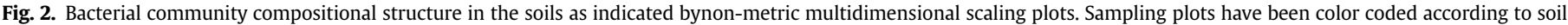
pH. BS:bulk soil; LS:loosely bound soil; TS:tightly bound soil.

(0.274), suggesting a more compact topology with more direct paths of communication between nodes due to its high transitivity pattern within tightly bound soil. In addition, the three investigated networks tended to have different degree distribution patterns (Fig. 3B). Bulk soil displayed a random distribution pattern for both OTU and class level nodes; loosely bound soil showed a random distribution for OTU level and a power-law distribution for class level; and tightly bound soil had a power-law distribution pattern (Bergman and Siegal, 2003) for both OTU and class level, indicating the hub-based topology for this network (Schüler and BornbergBauer, 2011). The nodes were further classified as peripheral, intermediate or central (Greenblum et al., 2012) according to their betweeness values for the total network at the both the OTU and Class level (Fig. S3). Intermediate nodes were more abundant in bulk soil while peripheral nodes were more abundant in the loosely bound and tightly bound soil, and tightly bound soil had the greatest proportion of nodes with central position (Fig. S3). We also analyzed the network properties of the dominant bacteria phyla, and found that Alphaproteobacteria and Betaproteobacteria, which were abundant, also had high degree and betweeness in all three compartments. However, Actinobacteria had highest relative abundance in all three compartments (Table S3), but had very low degree and betweenness (Table S14), while WS3 whose relative abundance was less than $1 \%$ (Table S3), had high degree and betweeness (Table S14).

\subsection{Distance decay patterns and assembly processes in bulk soil, loosely bound soil and tightly bound soil}

In order to examine the bacterial community distribution patterns over spatial distance among bulk soil, loosely bound soil and tightly bound soil, both compositional and phylogenetic distance decay were calculated (Fig. 4). We found a similar compositional distance decay relationships (with approximate equal decay rates) in bulk soil, loosely bound soil and tightly bound soil (Fig. 4A; Table S15). Meanwhile, we also identified a phylogenetic distance decay relationship whose decay rates were significantly higher in tightly bound soil by covariance analysis (Fig. 4B; Fig. S4; Table S15) suggesting that geographic distance played a relatively more important role than environmental variation in shaping bacterial community distribution in the tightly bound soil compared with the other compartments. Additionally, the significance of the relationship between community similarity, phylogenetic similarity with geographical distance and environmental dissimilarity was also assessed by partial mantel test (Table 1 ) and multiple regression with distance matrices (MRM) (Table S16). It showed that the local environmental factors had a greater contribution to variance in bacterial community similarity between bulk and loosely bound soil; whereas less contribution to variance in tightly bound soil.

To test whether dispersal limited or niche-based mechanisms best explain the assembly of the bacterial community in bulk soil, loosely bound soil and tightly bound soil, we calculated the NRI, NTI and NRI/NTI ratio (Fig. S5) for each single community, and betaNTI (beta nearest taxon index) for paired samples (Fig. 5). Increasingly positive NRI, NTI and NRI/NTI ratio scores indicate that cooccurring species are more phylogenetically related than expected by chance (phylogenetic clustering) (Hamilton et al., 2011). Our results (Fig. S5) demonstrated that most of NRI, NTI and NRI/ NTI scores were above 2 in all sampling plots, and their mean values were all positive, but significantly lower in tightly bound soil compared with loosely bound soil (NRI, $\mathrm{P}=0.012$; NTI, $\mathrm{P}=0.003$; NRI/NTI, P = 0.001; ANOVA) and bulk soil (NRI, P = 0.008; NTI, $\mathrm{P}=0.002 ; \mathrm{NRI} / \mathrm{NTI}, \mathrm{P}=0.001 ;$ ANOVA). BetaNTI is the number of standard deviations the observed betaMNTD presents from the mean of null distribution, a betaNTI $<-2$ or $>+2$ indicates less than or greater than expected phylogenetic turnover or pair-wised comparison than expected by chance (Stegen et al., 2012). Our result showed that majority of the betaNTI scores were below -2 (92.5\% in bulk soil; $97.1 \%$ in loosely bound soil; $94.1 \%$ in tightly bound soil) (Fig. 5), which indicated that deterministic processes governed community dynamics in all compartments. Meanwhile, some of the betaNTI scores (7.5\% in bulk soil; $2.9 \%$ in loosely bound soil; $5.9 \%$ in tightly bound soil) were included in the range of above -2 and below +2 , which were consistent with random phylogenetic turnover. These results indicated that bacterial community assemblage processes in bulk soil, loosely bound soil and 


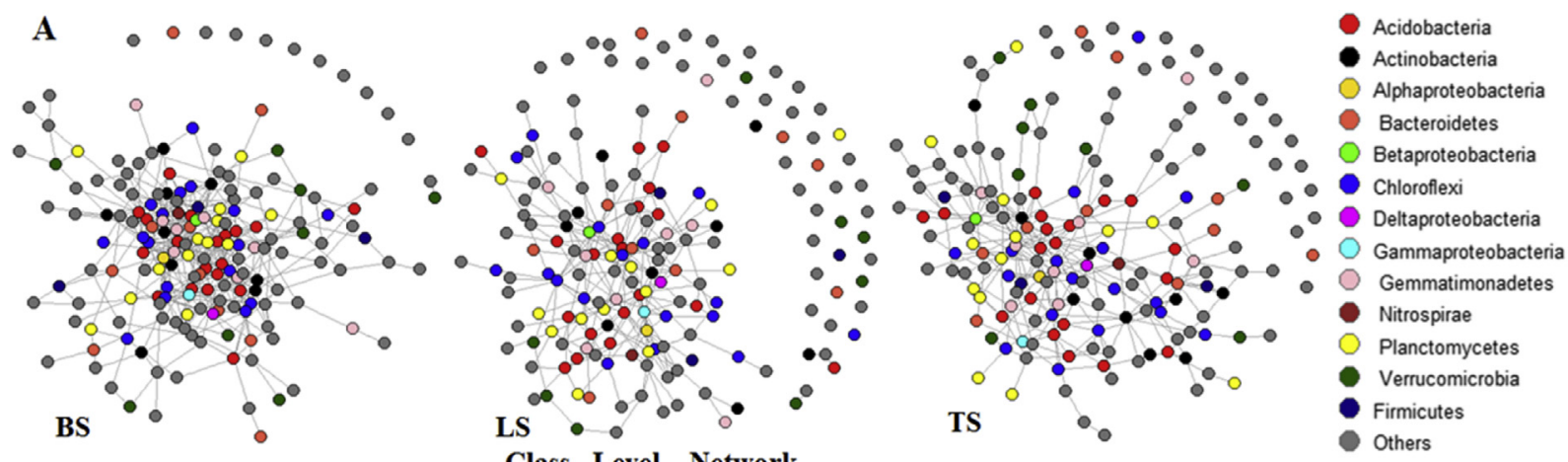

B

Class Level Network
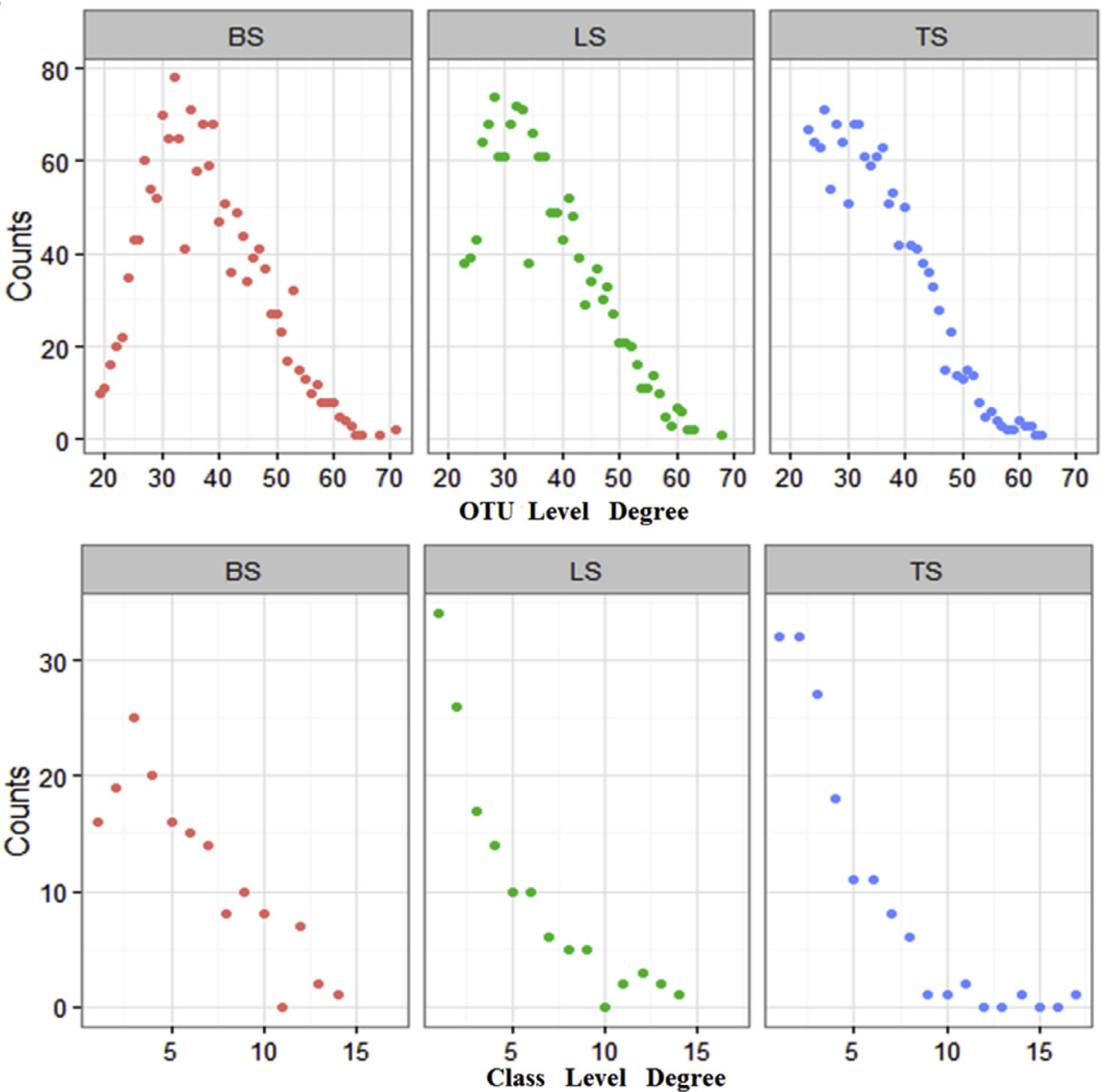

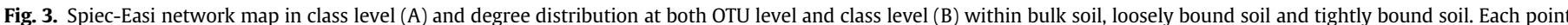
represents an independent bacterial class, edges show the relationship among classes. BS:bulk soil; LS:loosely bound soil; TS:tightly bound soil.

tightly bound soil tended to be deterministic, while phylogenetic clustering was weaker in tightly bound soil.

\section{Discussion}

4.1. Changes in soil $\mathrm{pH}$ describes less microbial variance in tightly bound soil compared to other soil compartments

As expected, soil $\mathrm{pH}$ was strongly correlated with the relative abundance of many phyla and families (Table S8, Table S9) and with alpha diversity (Table S10), which supports previous studies (Chu et al., 2010; Shen et al., 2013). However, in our study, it was less associated with the microbial distribution in tightly bound soil when compared to the other soil compartments, which could be due to the fact that $\mathrm{pH}$ was more stable in tightly bound soil in each sampling site (Table S2) compared to loosely bound and bulk soil as root-associated biogeochemistry could regulate $\mathrm{pH}$ fluctuations in this micro-environment (Dennis et al., 2010). Roots can regulate soil pH and change the buffering capacity of the rhizosphere soil (Wang et al., 2011); for example, Aspalathus linearis L. (rooibos tea bush) actively modifies its rhizosphere $\mathrm{pH}$ by exuding $\mathrm{OH}^{-}$and $\mathrm{HCO}_{3}^{-}$to facilitate growth in low pH soils (Mommer et al., 2016). Total phosphorus was also important in describing the community patterns in the rhizosphere environment (TableS7), and is positively correlated with bacterial taxa and diversity (Table S8; Table S9; Table S10). This is highly associated with $\mathrm{pH}$ gradient 
$\mathbf{A}$

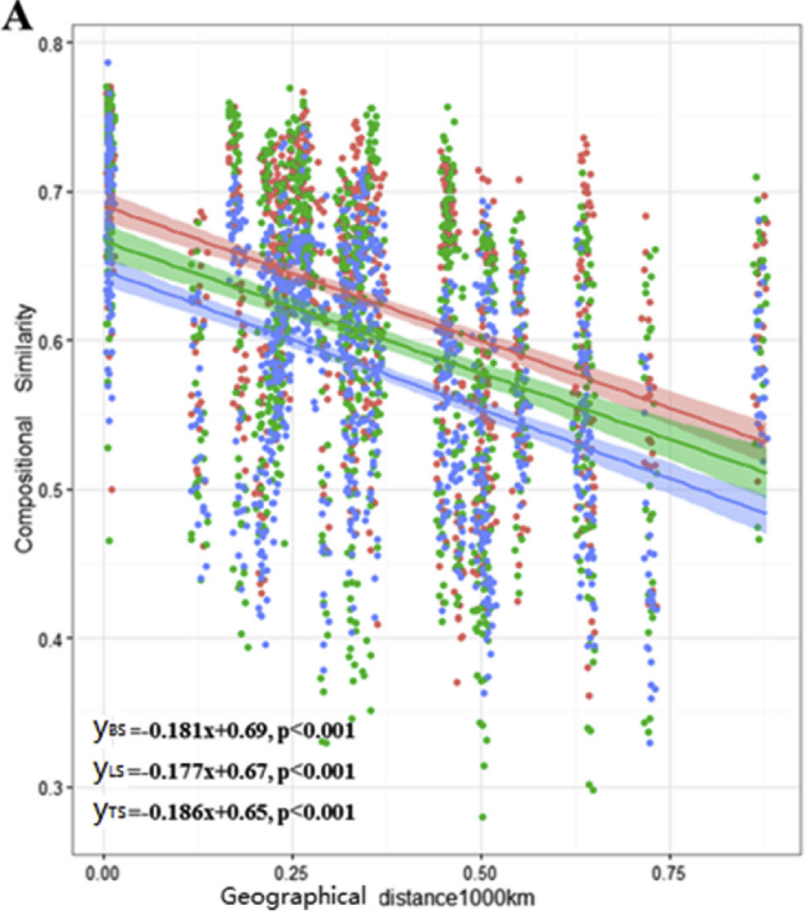

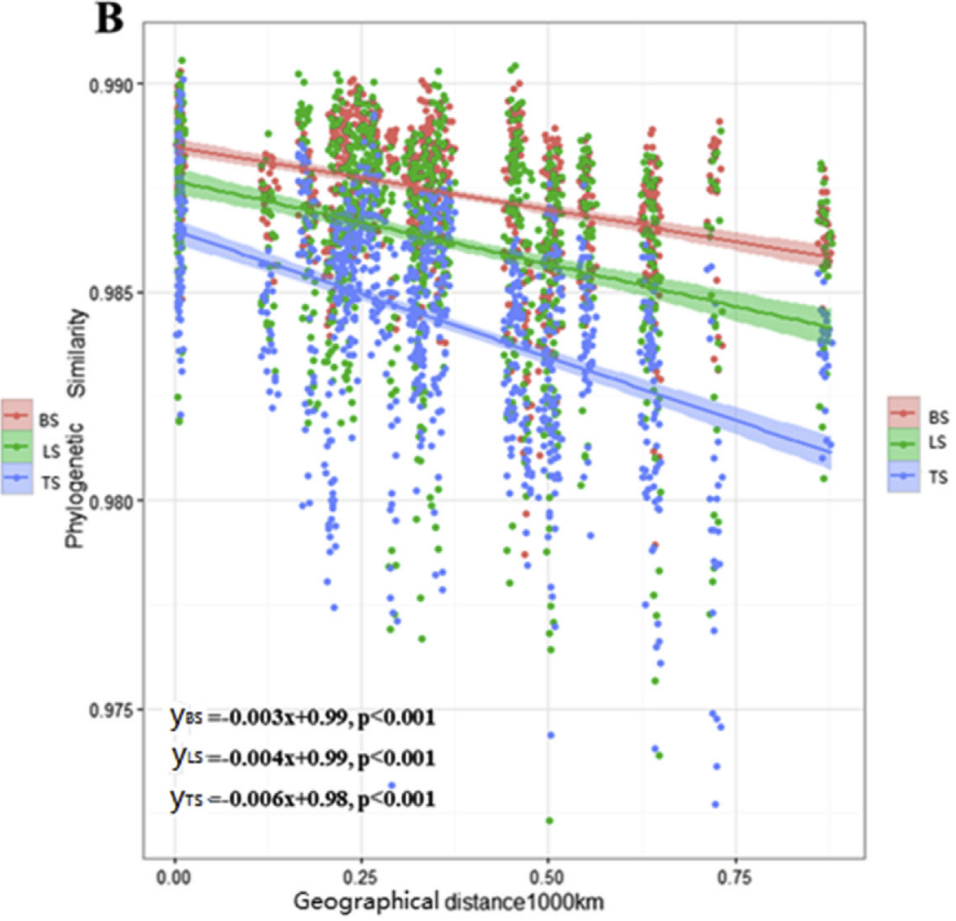

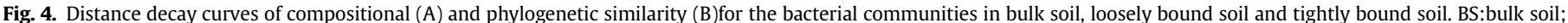
LS:loosely bound soil; TS:tightly bound soil.

Table 1

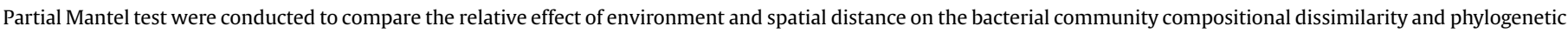
dissimilarity. BS: bulk soil; LS: loosely bound soil; TS: tightly bound soil.

\begin{tabular}{|c|c|c|c|c|}
\hline \multirow[t]{2}{*}{ Partial Mantel } & \multicolumn{2}{|l|}{ Compositional dissimilarity } & \multicolumn{2}{|l|}{ Phylogenetic dissimilarity } \\
\hline & Environment dissimilarity & Geographical distance & Environment dissimilarity & Geographical distance \\
\hline BS & $0.3165(0.002)$ & $0.2881(0.001)$ & $0.4443(0.001)$ & $0.1781(0.001)$ \\
\hline LS & $0.3576(0.001)$ & $0.2026(0.001)$ & $0.4538(0.001)$ & $0.1509(0.001)$ \\
\hline TS & $0.1667(0.002)$ & $0.3335(0.001)$ & $0.2692(0.001)$ & $0.3752(0.001)$ \\
\hline
\end{tabular}
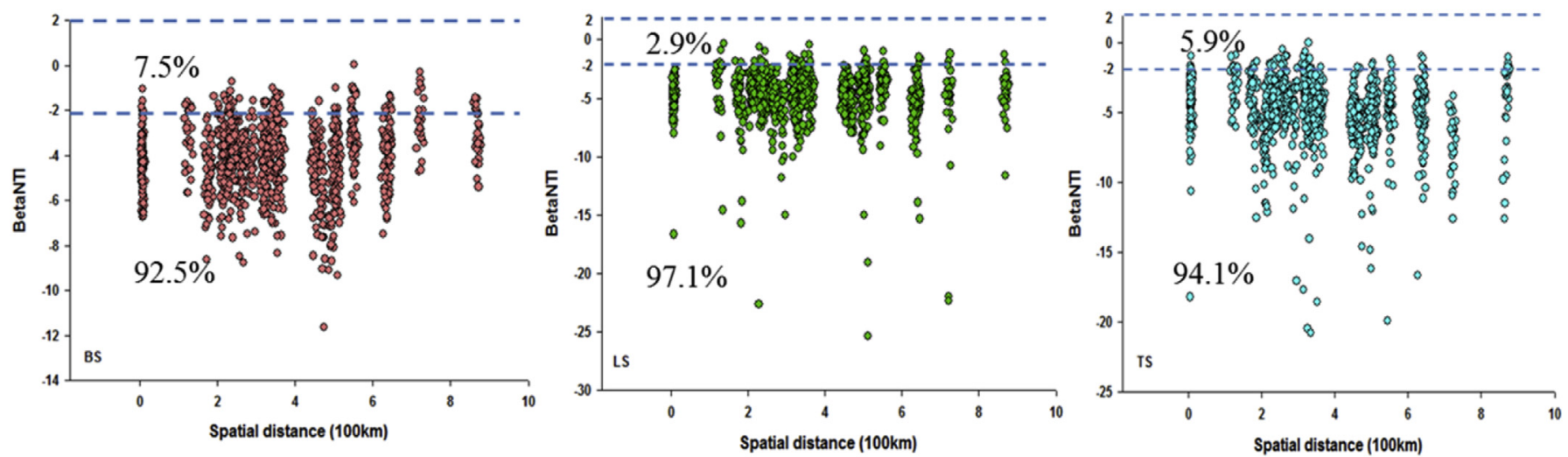

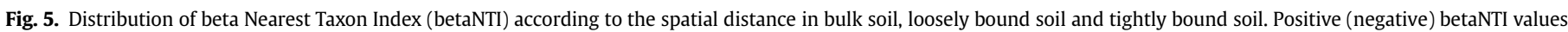

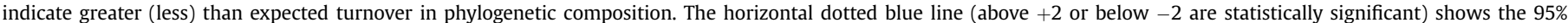
confidence intervals around the expectation under neutral community assembly. BS:bulk soil; LS:loosely bound soil; TS:tightly bound soil.

pattern, as soil organic phosphorus can be mineralized by the root, microbial-borne phosphatases (George et al., 2008), and phytases (Starnes et al., 2008). Meanwhile, root-induced changes in the rhizosphere include acidification (Zhu et al., 2005) or alkalization (Rose et al., 2009), through the release of organic acid anions which mobilize phosphorus by ligand exchange, ligand-induced dissolution and complexation of cations bound to phosphorus ( $\mathrm{Li}$ et al., 2004). 


\subsection{Roots enrich and filter bacterial communities in rhizospheric} soil

Similar to the studies in crops such as maize (Peiffer et al., 2013), rice (Edwards et al., 2015) and barley (Bulgarelli et al., 2015), Proteobacteria, Actinobacteria and Acidobacteria mostly dominated in our wheat field (Fig. 1A; Table S2). The decreased alpha diversity with root proximity was inferred to the result of the root "filtration effect" (Dibbern et al., 2014), whereby plants might select a microbiome in two steps. First, a general recruitment to the vicinity of the root and second, transition from external to internal occupancy in the root (Edwards et al., 2015). The selection of the bacterial community in the rhizosphere involves molecular signals from the plant, including components of root exudates and possibly cell-wall or membrane proteins (Turner et al., 2013; Bulgarelli et al., 2015; Edwards et al., 2015). In our study, tightly bound soil had a greater relative abundance of Actinobacteria (Table S3), which has the potential to produce a wide variety of antibiotics (Berdy, 2005; Bull et al., 2005), and therefore could possibly be involved in protecting roots from pathogenic bacteria. Alphaproteobacteria were also high enriched in tightly bound soil, which is consistent with the fast growth characteristics and with the ability to utilize a broad range of root-derived carbon substrates common to this Class (Lauber et al., 2009; Philippot et al., 2013). Acidobacteria, which decreased monotonically by soil pH changes (Bryant et al., 2008), were depleted in rhizospheric soil, especially tightly bound soil with reduced $\mathrm{pH}$ fluctuations. Plant roots select for specific microorganisms to colonize the rhizosphere (Berendsen et al., 2012), with the ultimate benefit of attracting and promoting beneficial microorganisms that can improve nutrient acquisition and combat pathogenic taxa (Dennis et al., 2010).

\subsection{Hub-based network structure in tightly bound soil}

The Spiec-Easi network analysis was set to see the associated properties of the three compartments, and it showed that bulk soil is more connected than other compartments, and loosely bound soil is probably the most dynamic community with a greater number of taxa with no common co-occurrence across sites. One explanation for this topological distinction is the niche differentiation (Ma et al., 2016) among bulk soil, loosely bound soil and tightly bound soil. When analyzing the network at the OTU level and the main sub-network at class level, we found that tightly bound soil was the least connected compartment; however, considering that the rhizosphere community is a subset of the bulk soil community, a less complex network is expected (Mendes et al., 2014). Totally, a hub-based structure (Arita, 2012; Rabbani et al., 2015) with higher transitivity (Table S9), a greater number of central nodes (Fig. S3) and power-law (Bergman and Siegal, 2003) distribution pattern was shown in tightly bound soil, which might be associated with the prompt need of high speed elements cycling (Hinsinger, 2001) and signal transmission (Whipps, 2001) in rhizosphere soil; however this needs to be tested in future studies. Additionally, Alphaproteobacteria, Betaproteobacteria, Detaproteobacteria and Gammaproteobacteria, which are generally considered r-strategists, had greater correlations with other species; one possible explanation is that these organisms require more cooperation with other bacteria when utilizing a broad range of root-derived carbon substrates (Lauber et al., 2009; Philippot et al., 2013). Although Actinobacteria had high relative abundance in all three compartments, it maintained a lower degree and betweeness (suggesting less connectivity) than other taxa; one possible explanation is that this group has the potential to produce antibiotics, thereby limiting potential interactions (Berdy, 2005; Bull et al., 2005). Conversely, WS3 had greater betweeness and degree than most other taxa in all three compartments, and this organism is known to degrade the polysaccharides from other bacteria (Youssef et al., 2015; Farag et al., 2017).

\subsection{Geographic distance contributed more in shaping bacterial community distribution pattern of tightly bound soil}

The compositional and phylogenetic distance decay relationships could help to understand, not only the shifts in compositional similarity with increasing distance, but also the phylogenetic nature of the shift (Bryant et al., 2008). In this study, we observed similar compositional distance decay patterns in three soil compartments which probably resulted from large number of OTUs (Fine and Kembel, 2011). Additionally, the phylogenetic distance decay turnover rate was greater in tightly bound soil compared with loosely bound soil and bulk soil, which coincided with the results of partial mantel test (Table 1) suggesting the greater contribution of geographical distance and relative less contribution of local environmental factors in shaping bacterial community distribution in tightly bound soil. We presume that with the addition of root derived products (Bais et al., 2006), the environment in tightly bound soil tended to be less variable when comparing with loosely bound soil and bulk soil, which resulted in relative less contribution of local environment factors in tightly bound soil.

\subsection{Deterministic assembly processes dominated in bulk and rhizospheric soil}

We identified that deterministic processes tended to dominate most of bacterial community assemblages in bulk soil, loosely bound soil and tightly bound soil. This result was different from the observation in a short-term (1-5 years) soybean cultivation study (Mendes et al., 2014), wherein microbial community structure demonstrated niche filtering in the rhizosphere, and neutral processes in bulk soil. Long-term cultivation may have promoted crops that select communities and functions that are beneficial to the plant, in that soil type, which follows niche-based theory (Mendes et al., 2014). In our study, the deterministic processes of bacterial community assemblage probably resulted from the long-term cultivation and artificial disturbance experienced by the wheat crop system (Jiang and Patel, 2008). In addition, we observed weaker phylogenetic clustering in tightly bound soil compared with the other two soil compartments. Chase (2010) proposed a stronger role for stochastic relative to deterministic assembly processes in higher resources environments (Chase, 2010). So we posit that root exudates from wheat (Bais et al., 2006) lead to localized increase in resources in tightly bound soil resulting in a weaker phylogenetic clustering effect.

In conclusion, bulk soil and rhizospheric soil maintained distinct bacterial community composition and diversity, which correlated with changes in soil pH. Bacterial communities in tightly bound soil had a greater hub-based network structure, which might be associated with rapid element cycling and signal transmission in the rhizosphere. Bacterial communities presented similar compositional, but different phylogenetic, distance decay patterns in bulk soil compared to rhizospheric soil, and geographical distance described the bacterial community spatial distribution better in tightly bound soil. Although deterministic processes dominated bacterial community assemblage in both bulk and rhizospheric soil, the community in tightly bound soil exhibited weaker phylogenetic clustering. These results expand our understanding of bacterial biogeography from bulk soil to rhizosphere in intensive agricultural ecosystems, which may provide the scientific basis for sustainable agriculture through biological process management. 


\section{Conflict of interest}

The authors declare no conflict of interest.

\section{Acknowledgements}

We thank Ruibo Sun, Kaoping Zhang, Dan He, Teng Yang and Yingying Ni for their assistance in soil sampling and lab analyses.This work was funded by the Strategic Priority Research Program of the Chinese Academy of Sciences (XDB15010101), the National Program on Key Basic Research Project (2014CB954002), the National Natural Science Foundation of China (41371254) and the National Key Research and Development Program of China (2017YFD0200604).

\section{Appendix A. Supplementary data}

Supplementary data related to this article can be found at http:// dx.doi.org/10.1016/j.soilbio.2017.06.020.

\section{References}

Arita, M., 2012. From metabolic reactions to networks and pathways. Bacterial Molecular Networks: Methods and Protocols 93-106.

Bais, H.P., Weir, T.L., Perry, L.G., Gilroy, S., Vivanco, J.M., 2006. The role of root exudates in rhizosphere interactions with plants and other organisms. Annual Review of Plant Biology 57, 233-266.

Berdy, J., 2005. Bioactive microbial metabolites. Journal of Antibiotics 58, 1.

Berendsen, R.L., Pieterse, C.M., Bakker, P.A., 2012. The rhizosphere microbiome and plant health. Trends in Plant Science 17, 478-486.

Bergman, A., Siegal, M.L., 2003. Evolutionary capacitance as a general feature of complex gene networks. Nature 424, 549-552.

Biddle, J.F., Fitz-Gibbon, S., Schuster, S.C., Brenchley, J.E., House, C.H., 2008. Metagenomic signatures of the Peru Margin subseafloor biosphere show a genetically distinct environment. Proceedings of the National Academy of Sciences $105,10583-10588$

Brink, S.C., 2016. Unlocking the secrets of the rhizosphere. Trends in Plant Science 3, $169-170$.

Bryant, J.A., Lamanna, C., Morlon, H., Kerkhoff, A.J., Enquist, B.J., Green, J.L., 2008. Microbes on mountainsides: contrasting elevational patterns of bacterial and plant diversity. Proceedings of the National Academy of Sciences 105, $11505-11511$.

Bulgarelli, D., Garrido-Oter, R., Munch, P.C., Weiman, A., Droge, J., Pan, Y. McHardy, A.C., Schulze-Lefert, P., 2015. Structure and function of the bacterial root microbiota in wild and domesticated barley. Cell Host Microbe 17, 392-403.

Bull, A.T., Stach, J.E., Ward, A.C., Goodfellow, M., 2005. Marine actinobacteria: perspectives, challenges, future directions. Antonie Van Leeuwenhoek 87, 65-79.

Caporaso, J.G., Kuczynski, J., Stombaugh, J., Bittinger, K., Bushman, F.D., Costello, E.K. Fierer, N., Pena, A.G., Goodrich, J.K., Gordon, J.I., 2010. QIIME allows analysis of high-throughput community sequencing data. Nature Methods 7, 335-336.

Cardona, C., Weisenhorn, P., Henry, C., Gilbert, J.A., 2016. Network-based metabolic analysis and microbial community modeling. Current Opinion in Microbiology 31, 124-131.

Carlson, K.M., Curran, L.M., Ratnasari, D., Pittman, A.M., Soares-Filho, B.S Asner, G.P., Trigg, S.N., Gaveau, D.A., Lawrence, D., Rodrigues, H.O., 2012 Committed carbon emissions, deforestation, and community land conversion from oil palm plantation expansion in West Kalimantan, Indonesia. Proceedings of the National Academy of Sciences 109, 7559-7564.

Caruso, T., Chan, Y., Lacap, D.C., Lau, M.C., McKay, C.P., Pointing, S.B., 2011. Stochastic and deterministic processes interact in the assembly of desert microbial communities on a global scale. The ISME Journal 5, 1406-1413.

Chase, J.M., 2010. Stochastic community assembly causes higher biodiversity in more productive environments. Science 328, 1388-1391,

Chen, J., Tang, C., Sakura, Y., Kondoh, A., Yu, J., Shimada, J., Tanaka, T., 2004. Spatial geochemical and isotopic characteristics associated with groundwater flow in the North China Plain. Hydrological Processes 18, 3133-3146.

Chu, H., Fierer, N., Lauber, C.L., Caporaso, J.G., Knight, R., Grogan, P., 2010. Soi bacterial diversity in the Arctic is not fundamentally different from that found in other biomes. Environmental Microbiology 12, 2998-3006.

Chu, H., Sun, H., Tripathi, B.M., Adams, J.M., Huang, R., Zhang, Y., Shi, Y., 2016. Bacterial community dissimilarity between the surface and subsurface soils equals horizontal differences over several kilometers in the western Tibetan Plateau. Environmental Microbiology 18, 1523-1533.

Dennis, P.G. Miller, A.J., Hirsch, P.R., 2010. Are root exudates more important than other sources of rhizodeposits in structuring rhizosphere bacterial communities? FEMS Microbiology Ecology 72, 313-327.

Dibbern, D., Schmalwasser, A., Lueders, T., Totsche, K.U., 2014. Selective transport of plant root-associated bacterial populations in agricultural soils upon snowmelt. Soil Biology and Biochemistry 69, 187-196.

Donn, S., Kirkegaard, J.A., Perera, G., Richardson, A.E., Watt, M., 2015. Evolution of bacterial communities in the wheat crop rhizosphere. Environmental Microbiology $17,610-621$

Dumbrell, A.J., Nelson, M., Helgason, T., Dytham, C., Fitter, A.H., 2010. Relative roles of niche and neutral processes in structuring a soil microbial community. The ISME Journal 4, 337-345.

Edgar, R.C., 2010. Search and clustering orders of magnitude faster than BLAST. Bioinformatics 26, 2460-2461.

Edwards, J., Johnson, C., Santos-Medellin, C., Lurie, E., Podishetty, N.K., Bhatnagar, S., Eisen, J.A., Sundaresan, V., 2015. Structure, variation, and assembly of the rootassociated microbiomes of rice. Proceedings of the National Academy of Sciences 112, E911-E920.

Farag, I.F., Youssef, N.H., Elshahed, M.S., 2017. Global distribution patterns and pangenomic diversity of the candidate phylum "Latescibacteria"(WS3). Applied and Environmental Microbiology, 00521-00517.

Ferrenberg, S., O'Neill, S.P., Knelman, J.E., Todd, B., Duggan, S., Bradley, D., Robinson, T., Schmidt, S.K., Townsend, A.R., Williams, M.W., 2013. Changes in assembly processes in soil bacterial communities following a wildfire disturbance. The ISME Journal 7, 1102-1111.

Fierer, N., Jackson, R.B., 2006. The diversity and biogeography of soil bacterial communities. Proceedings of the National Academy of Sciences 103, 626-631.

Fine, P.V., Kembel, S.W., 2011. Phylogenetic community structure and phylogenetic turnover across space and edaphic gradients in western Amazonian tree communities. Ecography 34, 552-565.

Fischer, R., Edmeades, G.O., 2010. Breeding and cereal yield progress. Crop Science 50. S-85-S-98.

Ge, Y., He, J.Z., Zhu, Y.G., Zhang, J.B., Xu, Z., Zhang, L.M., Zheng, Y.M., 2008. Differences in soil bacterial diversity: driven by contemporary disturbances or historical contingencies? The ISME Journal 2, 254-264.

George, T.S., Gregory, P.J., Hocking, P., Richardson, A.E., 2008. Variation in rootassociated phosphatase activities in wheat contributes to the utilization of organic P substrates in vitro, but does not explain differences in the P-nutrition of plants when grown in soils. Environmental and Experimental Botany 64, 239-249.

Greenblum, S., Turnbaugh, P.J., Borenstein, E., 2012. Metagenomic systems biology of the human gut microbiome reveals topological shifts associated with obesity and inflammatory bowel disease. Proceedings of the National Academy of Sciences 109, 594-599.

Griffiths, R.I. Thomson, B.C. James, P. Bell, T., Bailey, M., Whiteley, A.S. 2011. The bacterial biogeography of British soils. Environmental Microbiology 13, $1642-1654$.

Gumiere, T., Durrer, A., Bohannan, B.J., Andreote, F.D., 2016. Biogeographical patterns in fungal communities from soils cultivated with sugarcane. Journal of Biogeography 43.

Högberg, M.N., Högberg, P., Myrold, D.D., 2007. Is microbial community composition in boreal forest soils determined by pH, C-to-N ratio, the trees, or all three? Oecologia 150, 590-601.

Hamilton, T.L., Boyd, E.S., Peters, J.W., 2011. Environmental constraints underpin the distribution and phylogenetic diversity of nifH in the Yellowstone geothermal complex. Microbial Ecology 61, 860-870.

Hardy, O.J., 2008. Testing the spatial phylogenetic structure of local communities: statistical performances of different null models and test statistics on a locally neutral community. Journal of Ecology 96, 914-926.

Hinsinger, P., 2001. Bioavailability of soil inorganic P in the rhizosphere as affected by root-induced chemical changes: a review. Plant and Soil 237, 173-195.

Huse, S.M., Huber, J.A., Morrison, H.G., Sogin, M.L., Welch, D.M., 2007. Accuracy and quality of massively parallel DNA pyrosequencing. Genome Biology 8, R143.

Jiang, L., Patel, S.N., 2008. Community assembly in the presence of disturbance: a microcosm experiment. Ecology 89, 1931-1940.

Kennedy, A., Smith, K., 1995. Soil microbial diversity and the sustainability of agricultural soils. Plant and Soil 170, 75-86.

Kurtz, Z.D., Müller, C.L., Miraldi, E.R., Littman, D.R., Blaser, M.J., Bonneau, R.A., 2015. Sparse and compositionally robust inference of microbial ecological networks. PLoS Computational Biology 11, e1004226.

Lauber, C.L., Hamady, M., Knight, R., Fierer, N., 2009. Pyrosequencing-based assessment of soil $\mathrm{pH}$ as a predictor of soil bacterial community structure at the continental scale. Applied and Environmental Microbiology 75, 5111-5120.

Li, S.M., Li, L., Zhang, F.S., Tang, C., 2004. Acid phosphatase role in chickpea/maize intercropping. Annals of Botany 94, 297-303.

Liu, J., Sui, Y., Yu, Z., Shi, Y., Chu, H., Jin, J., Liu, X., Wang, G., 2014. High throughput sequencing analysis of biogeographical distribution of bacterial communities in the black soils of northeast China. Soil Biology and Biochemistry 70, 113-122.

Liu, J., Sui, Y., Yu, Z., Yao, Q., Shi, Y., Chu, H., Jin, J., Liu, X., Wang, G., 2016. Diversity and distribution patterns of acidobacterial communities in the black soil zone of northeast China. Soil Biology and Biochemistry 95, 212-222.

Ma, B., Wang, H., Dsouza, M., Lou, J., He, Y., Dai, Z., Brookes, P.C., Xu, J., Gilbert, J.A., 2016. Geographic patterns of co-occurrence network topological features for soil microbiota at continental scale in eastern China. The ISME Journal 10, 1891-1901.

Martiny, J.B., Eisen, J.A., Penn, K., Allison, S.D., Horner-Devine, M.C., 2011. Drivers of bacterial $\beta$-diversity depend on spatial scale. Proceedings of the National Academy of Sciences 108, 7850-7854.

Mendes, L.W., Kuramae, E.E., Navarrete, A.A., van Veen, J.A., Tsai, S.M., 2014. 
Taxonomical and functional microbial community selection in soybean rhizosphere. The ISME Journal 8, 1577-1587.

Mommer, L., Kirkegaard, J., van Ruijven, J., 2016. Root-root interactions: towards a rhizosphere framework. Trends in Plant Science 21, 209-217.

Nacke, H., Thürmer, A., Wollherr, A., Will, C., Hodac, L., Herold, N., Schöning, I. Schrumpf, M., Daniel, R., 2011. Pyrosequencing-based assessment of bacterial community structure along different management types in German forest and grassland soils. PLoS One 6, e17000.

Nekola, J.C., White, P.S., 1999. The distance decay of similarity in biogeography and ecology. Journal of Biogeography 26, 867-878.

Ofiţeru, I.D., Lunn, M., Curtis, T.P., Wells, G.F., Criddle, C.S., Francis, C.A., Sloan, W.T., 2010. Combined niche and neutral effects in a microbial wastewater treatment community. Proceedings of the National Academy of Sciences 107, $15345-15350$.

Peiffer, J.A., Spor, A., Koren, O., Jin, Z., Tringe, S.G., Dangl, J.L., Buckler, E.S., Ley, R.E., 2013. Diversity and heritability of the maize rhizosphere microbiome under field conditions. Proceedings of the National Academy of Sciences 110, 6548-6553.

Philippot, L., Raaijmakers, J.M., Lemanceau, P., van der Putten, W.H., 2013. Going back to the roots: the microbial ecology of the rhizosphere. Nature Reviews Microbiology 11, 789-799.

Purcell, D., Sompong, U., Yim, L.C., Barraclough, T.G., Peerapornpisal, Y., Pointing, S.B., 2007. The effects of temperature, $\mathrm{pH}$ and sulphide on the community structure of hyperthermophilic streamers in hot springs of northern Thailand. FEMS Microbiology Ecology 60, 456-466.

Rabbani, M., Farshbaf-Geranmayeh, A., Hasani, M., Rezaei, M., 2015. A variable neighborhood search algorithm for network expansion deferment in a hub network. International Journal of Strategic Decision Sciences (IJSDS) 6, 17-32.

Rose, T.J., Hardiputra, B., Rengel, Z., 2009. Wheat, canola and grain legume access to soil phosphorus fractions differs in soils with contrasting phosphorus dynamics. Plant and Soil 326, 159-170.

Saito, V.S., Soininen, J., Fonseca-Gessner, A.A., Siqueira, T., 2015. Dispersal traits drive the phylogenetic distance decay of similarity in neotropical stream metacommunities. Journal of Biogeography 42, 2101-2111.

Schüler, A., Bornberg-Bauer, E., 2011. The evolution of protein interaction networks. Data Mining in Proteomics: From Standards to Applications 273-289.

Shen, C., Xiong, J., Zhang, H., Feng, Y., Lin, X., Li, X., Liang, W., Chu, H., 2013. Soil pH drives the spatial distribution of bacterial communities along elevation on Changbai Mountain. Soil Biology and Biochemistry 57, 204-211.

Starnes, D.L., Padmanabhan, P., Sahi, S.V., 2008. Effect of P sources on growth, P accumulation and activities of phytase and acid phosphatases in two cultivars of annual ryegrass (Lolium multiflorum L.). Plant Physiology and Biochemistry $46,580-589$

Stegen, J.C., Lin, X., Konopka, A.E., Fredrickson, J.K., 2012. Stochastic and deterministic assembly processes in subsurface microbial communities. The ISME Journal 6, 1653-1664.

Turner, T.R., Ramakrishnan, K., Walshaw, J., Heavens, D., Alston, M., Swarbreck, D. Osbourn, A., Grant, A., Poole, P.S., 2013. Comparative metatranscriptomics reveals kingdom level changes in the rhizosphere microbiome of plants. The ISME Journal 7, 2248-2258.

Wang, Y., Marschner, P., Zhang, F., 2011. Phosphorus pools and other soil properties in the rhizosphere of wheat and legumes growing in three soils in monoculture or as a mixture of wheat and legume. Plant and Soil 354, 283-298.

Wang, Z., Shan, X.-q., Zhang, S., 2002. Comparison between fractionation and bioavailability of trace elements in rhizosphere and bulk soils. Chemosphere 46 , 1163-1171.

Webb, C.O., 2000. Exploring the phylogenetic structure of ecological communities: an example for rain forest trees. The American Naturalist 156, 145-155.

Whipps, J.M., 2001. Microbial interactions and biocontrol in the rhizosphere. Journal of Experimental Botany 52, 487-511.

Will, C., Thürmer, A., Wollherr, A., Nacke, H., Herold, N., Schrumpf, M., Gutknecht, J., Wubet, T., Buscot, F., Daniel, R., 2010. Horizon-specific bacterial community composition of German grassland soils, as revealed by pyrosequencing-based analysis of 16S rRNA genes. Applied and Environmental Microbiology 76, $6751-6759$.

Youssef, N.H., Farag, I.F., Rinke, C., Hallam, S.J., Woyke, T., Elshahed, M.S., 2015. In silico analysis of the metabolic potential and niche specialization of candidate phylum "Latescibacteria" (WS3). PLoS One 10, e0127499.

Zarraonaindia, I., Owens, S.M., Weisenhorn, P., West, K., Hampton-Marcell, J., Lax, S. Bokulich, N.A., Mills, D.A., Martin, G., Taghavi, S., 2015. The soil microbiome influences grapevine-associated microbiota. MBio 6 e02527-02514.

Zhou, J., Deng, Y., Shen, L., Wen, C., Yan, Q., Ning, D., Qin, Y., Xue, K., Wu, L., He, Z. Voordeckers, J.W., Nostrand, J.D., Buzzard, V., Michaletz, S.T., Enquist, B.J. Weiser, M.D., Kaspari, M., Waide, R., Yang, Y., Brown, J.H., 2016. Temperature mediates continental-scale diversity of microbes in forest soils. Nature Communications 7, 12083.

Zhu, Y., Yan, F., Zorb, C., Schubert, S., 2005. A link between citrate and proton release by proteoid roots of white lupin (Lupinus albus L.) grown under phosphorusdeficient conditions? Plant and Cell Physiology 46, 892-901. 